Theoret. chim. Acta (Berl.) 1, 468 (1963)

Department of Chemistry, Texas Technological College, Lubbock, Texas

\title{
Erratum
}

\section{Studies of Delocalized Electron Bonding}

Theoret. chim. Acta (Berl.) 1, 133, 222 (1963)

By

\section{HARRY G. HECHT}

An error has been noted in the solution of the secular equations affecting the calculations of paper I [Theoret. chim. Acta (Berl.) 1, 133 (1963)] and paper II [Theoret. chim. Acta (Berl.) 1, 222 (1963)]. Tab. 2 of paper I should read:

\begin{tabular}{r|c|c|c|c|c|c}
\hline$\varphi$ & $E$ (Kcal/mole) & $c_{1}$ & $c_{2}$ & $c_{3}$ & $c_{4}, c_{5}$ & $J_{H H^{\prime}}$ (c.p.s.) \\
\hline $0^{\circ}$ & -278.6857 & 0.99059 & 0.05748 & -0.00310 & -0.02008 & 26.84 \\
$15^{\circ}$ & -278.8212 & 0.99113 & 0.05650 & -0.00300 & -0.02012 & 26.38 \\
$30^{\circ}$ & -279.1855 & 0.99260 & 0.05382 & -0.00272 & -0.02021 & 25.13 \\
$45^{\circ}$ & -279.6730 & 0.99471 & 0.04996 & -0.00233 & -0.02034 & 23.35 \\
$60^{\circ}$ & -280.1202 & 0.99721 & 0.04535 & -0.00187 & -0.02050 & 21.21 \\
$75^{\circ}$ & -280.4003 & 0.99989 & 0.04037 & -0.00138 & -0.02066 & 18.92 \\
$90^{\circ}$ & -280.4166 & 1.00255 & 0.03541 & -0.00089 & -0.02083 & 16.64 \\
$105^{\circ}$ & -280.1771 & 1.00524 & 0.03035 & -0.00039 & -0.02100 & 14.33 \\
$120^{\circ}$ & -279.7019 & 1.00774 & 0.02562 & 0.00009 & -0.02116 & 12.18 \\
$135^{\circ}$ & -279.1349 & 1.01003 & 0.02127 & 0.00053 & -0.02131 & 10.22 \\
$150^{\circ}$ & -278.5886 & 1.01185 & 0.01781 & 0.00089 & -0.02143 & 8.66 \\
$165^{\circ}$ & -278.1997 & 1.01305 & 0.01551 & 0.00113 & -0.02152 & 7.62 \\
$180^{\circ}$ & -278.0577 & 1.01346 & 0.01474 & 0.00121 & -0.02154 & 7.28 \\
& & & & & &
\end{tabular}

These corrected solutions lead to a calculated rotation barrier of $0.63 \mathrm{Kcal} / \mathrm{mole}$ favoring the staggered configuration. The $\delta$ integral for $105^{\circ}$ listed in Tab. 1 of Paper I was erroneously listed as +4.78 rather than +3.78 .

Equations (1), III, paper II, are,

III: $W=-16.6843$ e.v.;

$\left.\Psi=1.0818 \psi_{a b, c d, e f, g h-0.0278\left(\psi_{a b}, c h, d g, e f\right.}+\psi_{a f, b e, c d, g h}\right)$

$-0.0286\left(\psi_{a h, b g, c d, e f}+\psi_{a b, c d, e h, f g}+\psi_{a d, b c, e f, g h}+\psi_{a b, c f}, d e, g h\right)$

$+0.0008\left(\psi_{a h, b c, d g, e f}+\psi_{a b, c h, d e, f g}+\psi_{a f, b c, d e, g h}+\psi_{a h, b e, c d, f g}\right)$

$-0.0016 \psi_{a h, b c, d e, f g}+0.0016\left(\psi_{a h, b g}, c f, a e+\psi_{a d}, b c, e h, f g\right)$,

and the last row of the table in Paper II should read:

\begin{tabular}{c|c|c|c|c|c|c}
\hline $\begin{array}{c}\text { Calcu- } \\
\text { lation }\end{array}$ & $\begin{array}{c}\text { Number of } \\
\text { Eleetrons }\end{array}$ & $\begin{array}{c}\text { Number of } \\
\text { v. b. Structures }\end{array}$ & $\begin{array}{c}\Delta W_{\text {res }} \\
\text { (e. v.) }\end{array}$ & $\begin{array}{c}J_{H H^{\prime}} \\
\text { (c. p. s.) }\end{array}$ & $\eta_{H H^{\prime} \text { (exchange }}$ order) & $\begin{array}{c}P_{H H^{\prime}} \\
\text { (bond order) }\end{array}$ \\
\hline III & 8 & 14 & 0.0563 & 12.55 & -0.4595 & 0.0270
\end{tabular}

These corrections do not substantially alter the discussion and conclusions of either Paper I or II. 